\title{
Safety climate, safety climate strength, and length of stay in the NICU
}

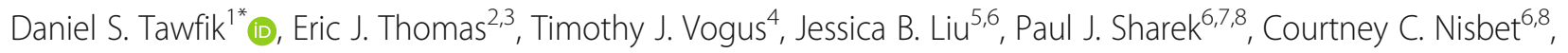
Henry C. Lee ${ }^{5,6}$, J. Bryan Sexton ${ }^{9,10}$ and Jochen Profit ${ }^{5,6}$

\begin{abstract}
Background: Safety climate is an important marker of patient safety attitudes within health care units, but the significance of intra-unit variation of safety climate perceptions (safety climate strength) is poorly understood. This study sought to examine the standard safety climate measure (percent positive response (PPR)) and safety climate strength in relation to length of stay (LOS) of very low birth weight (VLBW) infants within California neonatal intensive care units (NICUs).

Methods: Observational study of safety climate from 2073 health care providers in 44 NICUs. Consistent perceptions among a NICU's respondents, i.e., safety climate strength, was determined via intra-unit standard deviation of safety climate scores. The relation between safety climate PPR, safety climate strength, and LOS among VLBW $(<1500 \mathrm{~g})$ infants was evaluated using log-linear regression. Secondary outcomes were infections, chronic lung disease, and mortality.

Results: NICUs had safety climate PPRs of $66 \pm 12 \%$, intra-unit standard deviations 11 (strongest) to 23 (weakest), and median LOS 60 days. NICUs with stronger climates had LOS 4 days shorter than those with weaker climates. In interaction modeling, NICUs with weak climates and low PPR had the longest LOS, NICUs with strong climates and low PPR had the shortest LOS, and NICUs with high PPR (both strong and weak) had intermediate LOS. Stronger climates were associated with lower odds of infections, but not with other secondary outcomes.

Conclusions: Safety climate strength is independently associated with LOS and moderates the association between PPR and LOS among VLBW infants. Strength and PPR together provided better prediction than PPR alone, capturing variance in outcomes missed by PPR. Evaluations of NICU safety climate consider both positivity (PPR) and consistency of responses (strength) across individuals.
\end{abstract}

Keywords: Safety climate, Safety climate strength, Length of stay, Quality of care, Neonatology

\section{Background}

Error reduction in health care relies on a shared understanding that the organization prioritizes safety behaviors and practices above competing interests, and that it recognizes, rewards, and supports such behaviors by all employees. $(1,2)$ In other words, it relies on a welldeveloped safety climate. In order to achieve this, two separate but related elements are required. First, the actions of organizational leaders must emphasize safety in an obvious way. Second, the workforce must largely

\footnotetext{
* Correspondence: dtawfik@stanford.edu

${ }^{1}$ Division of Pediatric Critical Care Medicine, Department of Pediatrics,

Stanford University School of Medicine, 770 Welch Road, Suite 435, Stanford, CA 94304, USA

Full list of author information is available at the end of the article
}

agree in its assessment of the emphasis on safety. Workforce assessments of patient safety climate are typically measured through safety climate surveys, which have been used extensively in health care research. (3-15)

Ample research suggests that a more positive safety climate is associated with a host of workforce and patient outcomes. Correspondingly, safety climate is increasingly being understood as a driver of quality of care, $(2,6,12$, $14,16-19)$ and benchmarking of safety climate is now a focus in the United States by the Joint Commission, (20) the Agency for Healthcare Research and Quality, (21) and Leapfrog. (22) However, some recent work also suggests (albeit with low response rates) that safety climate may not be a key mechanism underpinning safety and is

(c) The Author(s). 2019 Open Access This article is distributed under the terms of the Creative Commons Attribution 4.0 International License (http://creativecommons.org/licenses/by/4.0/), which permits unrestricted use, distribution, and reproduction in any medium, provided you give appropriate credit to the original author(s) and the source, provide a link to the Creative Commons license, and indicate if changes were made. The Creative Commons Public Domain Dedication waiver (http://creativecommons.org/publicdomain/zero/1.0/) applies to the data made available in this article, unless otherwise stated. 
otherwise difficult to shape. (23) Failures to modify safety climate may be a function of how it is assessed and interpreted. That is, prior research has focused on the level of safety climate (i.e., how positive it is) and largely assumed consistency.

However, safety climate perceptions may range from highly consistent to highly variable within an organization, with the degree of consistency termed safety climate strength. $(2,24)$ Climate strength derives from foundational psychological work by Walter Mischel which finds that situations differ in their ambiguity and "situational strength," with strong situations creating clarity that leads individuals with shared experiences to perceive events similarly and have uniform expectations regarding the most appropriate behaviors. (25) In contrast, weak situations are highly ambiguous, individuals have different perceptions, and inconsistent or non-existent behavioral expectations arise. Thus, when perceptions of the level of safety climate are consistent (i.e., strong safety climate), the priority placed on safety and the attendant behavioral expectations are clear, and more uniform behavior is likely to result. ${ }^{2,41}$ Additionally, climate strength also increases over time with strong climates persisting, while weaker ones may not. ${ }^{41}$ Therefore, the relationship between safety climate and outcomes is expected to be enhanced by a stronger safety climate. The more consistent the workforce safety experience is, the more likely it is to behave consistently as a collective with regard to safety. (26)

Since the Institute of Medicine report highlighting medical errors in 1999, (27) substantial efforts have focused on the reduction of medical errors in the United States. In parallel, there has also been a focus, particularly in the NICU, on the costs of preterm birth, with mean in-hospital costs of $\$ 76,000$ to $\$ 159$, 000 (in 2018 dollars) per very low birth weight (VLBW) infant. (28) Length of stay (LOS) carries particular importance as a quality measure for VLBW infants, as it serves multiple roles: a marker of value for health policy makers and payers, a competitive benchmark for payment, a measure of quality and family centered care, an indicator of safety lapses, and a target for quality improvement. (29) Consequently, there is great interest by hospitals operating NICUs and health policy makers alike to deliver safer care in ways that also reduce LOS.

Although safety climate levels vary among NICUs, $(10,30,31)$ safety climate strength profiles of NICUs are unexamined, and the relation between safety climate strength and neonatal quality of care is unknown. Thus, the objective of this study was to analyze the direct and interactive relationships between safety climate and safety climate strength in relation to LOS, with exploratory analysis of other related outcomes among VLBW $(<1500 \mathrm{~g})$ infants.

\section{Methods}

This cross-sectional study links caregiver perceptions of safety climate to clinical outcomes data derived from a population-based clinical registry among 44 California NICUs.

\section{Sample and procedure Selection of NICUs}

A cross-sectional survey of safety climate and workforce engagement was offered to a voluntary sample of NICUs participating in a quality improvement initiative organized by the California Perinatal Quality Care Collaborative (CPQCC). (32) Of the 61 NICUs who participated in the improvement initiative, 44 participated in the survey, which was administered at the beginning of the improvement initiative (between June and September 2011).

Staff with a 0.5 full time equivalent or greater time commitment to the NICU for at least the four consecutive weeks prior to survey administration were eligible for inclusion. Paper-based surveys were administered during routine departmental and staff meetings. Respondents returned surveys to a locked box or sealable envelope to maintain confidentiality. Individuals not present in routine meetings were hand-delivered a survey, pencil, and return envelope. This administration technique has generated high response rates $(13,33)$ comparable to other studies of similar methodology. (34) CPQCC administered the survey and transmitted a de-identified data set to the authors for analysis.

\section{Selection of patients}

In order to capture outcomes concurrent with and subsequent to survey responses, clinical data routinely submitted to the CPQCC by Collaborative members reflecting VLBW infants born between January 1, 2011 and December 31, 2013 were linked to the survey data using unique identifiers for NICUs and patients. We used multiyear analysis due to the small number of VLBW infants cared for in some institutions.

\section{Measures \\ Survey data}

For this study, we used the 7-item safety climate scale of the Safety Attitudes Questionnaire (SAQ) (13), scored for each individual on a $0-100$ scale. For each NICU, the proportion of respondents achieving a score $\geq 75$ out of 100 was calculated and reported as the percent positive response (PPR), in line with prior research. (35)

In addition, distribution of individual safety climate scores was plotted for each NICU, and summary statistics calculated, including mean, median, standard deviation (SD), and $r_{\mathrm{wg}(\mathrm{j})}$ (a measure of agreement, often used in inter-rater reliability assessments). The SD of individual safety climate scores within each NICU was 
used as the primary determination of its safety climate strength, in keeping with prior research. $(24,36,37)$

The survey also captured respondent characteristics including job position, years in specialty, gender, and predominant work shift. Job positions consisted of attending physicians, fellow physicians, neonatal nurse practitioners, registered nurses, and respiratory care practitioners.

\section{Clinical data}

CPQCC prospectively collects clinical data for infants born at 136 member hospitals using standard definitions developed by the Vermont Oxford Network, (38) and all data undergo a series of quality checks to ensure completeness and accuracy. Our primary outcome of interest was LOS. In line with other studies, $(39,40)$ we also evaluated post-menstrual age at discharge (PMA-DC) as a marker of LOS due to its closer approximation of a normal distribution and inherent adjustment for gestational age. LOS and PMA-DC were adjusted according to a prediction model developed in a previous study. (29) Covariates included sex, gestational age at birth, 5 min Apgar score (categorized as $<4,4-6$, or $>6$ ), small for gestational age ( $<10$ th percentile), birth at the NICU under investigation (inborn) or outborn, birth weight, maternal race, and binary variables representing antenatal steroid use, fetal distress, major anomalies, and maternal hypertension.

Infants who died prior to discharge were excluded from the LOS analysis, but included for secondary outcomes. This is because early deaths result in short LOS, but cannot be considered a positive outcome. Furthermore, the LOS associated with death can vary widely with different clinical trajectories. Prior work has shown that mortality rates are not correlated with LOS among survivors, suggesting that this exclusion is unlikely to bias our results. (29)

We also calculated the secondary clinical outcomes of health care-associated infection (HAI), chronic lung disease (CLD), and mortality using standard CPQCC definitions. HAI includes any bacterial or fungal infection acquired after 3 days of age during the birth hospitalization. For infants transferred to another facility, attribution of infection was defined to include those acquired "here" and "here and elsewhere." CLD is defined as oxygen requirement at 36 weeks post-menstrual age. We adjusted each secondary outcome according to a severity of illness model developed in a previous study. (35) Covariates included sex, gestational age at birth, 5 min Apgar score, small for gestational age (< 10th percentile), and birth at the NICU of interest (inborn) or outborn.

\section{Data analyses}

Descriptive statistics including frequencies, means, and SDs were used to describe survey responses and respondent demographics. Safety climate measures were calculated as described above, resulting in a safety climate PPR and safety climate strength for each NICU.

Following prior work on climate strength, $(36,37$, 41) we explored the relationship between safety climate strength and safety climate level. Because safety climate strength and PPR may be mathematically related, as cautioned by Bliese and Halverson (42) and Schneider et al., (36) we calculated the Pearson's correlation coefficient between the two measures, and plotted the relationships between strength and PPR for all NICUs. We evaluated the collinearity of strength and PPR as raw predictors and weighted predictors using variance inflation factors and the weighted correlation matrix, respectively.

To evaluate scale performance and to justify data aggregation to the NICU level of analysis, we calculated Cronbach's alpha, the intraclass correlation coefficients ICC (1) and ICC (2), as well as the average $r_{w g(j)}$ among all items. Cronbach's alpha is a measure of scale reliability (acceptable range $>0.7$ ). ICC (1) reflects the reliability of each individual provider's assessment of their NICU's mean safety climate (typical range $0.05-0.30$ ), and ICC (2) reflects the reliability of unit means and thus the ability to distinguish among NICUs based on individuals' responses (acceptable range $>0.7$ ). (43) $R_{w g(j)}$ is calculated at the NICU level and measures the degree to which individual responses within a NICU are consistent (acceptable range $>0.7$ ). (44)

Basic descriptive statistics examined the variation in LOS and clinical outcomes across NICUs. We used infant-level ordinary least squares models and logistic regression models for associations of safety climate measures with risk-adjusted LOS and clinical outcomes. (35) Models also included covariates for California Children's Services (CCS) level and birth year. Safety climate and safety climate strength terms were sequentially added to the models in the following order to illustrate any incremental effects of each factor: Safety climate PPR (Model 1), Safety climate PPR and safety climate strength (Model 2), and Safety climate PPR, safety climate strength, and the interaction between the two (Model 3). We used cluster-robust standard errors in all regressions, clustering by NICU. Analyses of LOS were performed using a log-normal marginal distribution due to its right-skewed distribution, and the Duan smearing retransformation (45) was used to obtain adjusted estimates from logtransformed regressions.

All statistical analyses were performed using SAS version 9.4. The study was approved by the Institutional Review Board at the first author's university with waiver of informed consent. 


\section{Results}

\section{Descriptive characteristics}

Forty-four NICUs participated in this study, with 2073 of 3294 surveys returned for a $62.9 \%$ response rate. Individual NICU response rates averaged 69.7\% (SD 19.8, range $22-100 \%)$, safety climate PPRs ranged from 33 to 95\% (mean 65.9 \pm 11.7, median 66.3, IQR 58.2-72.2), and safety climate strength ranged from 10.6 to 22.8 (mean $16.6 \pm 3.1$ ). Safety climate strength and PPR were positively correlated, as shown in Fig. $1 \quad(r=0.61$, $P<.001)$, but with acceptable variance inflation factors of 1.57 and 1.58, respectively, in multivariable modeling.

Table 1 lists respondent characteristics, which indicated $60 \%$ of all respondents with 11 or more years in their specialty, and $2.4 \%$ of all respondents with less than 1 year of experience. Across all respondents, the mean safety climate score was $76.5 \pm 17.5$, with $63.6 \%$ of respondents meeting the threshold score of $75 / 100$. The safety climate score distributions of representative NICUs with stronger and weaker climates are shown in Additional file 1: Figure S1.

\section{Scale performance and data aggregation}

Cronbach's alpha for the 7-item safety climate scale was 0.81 . ICC (1) was 0.06 and ICC (2) was 0.75 , suggesting that data aggregation at the level of the NICU is appropriate. (43) Similarly, the average $\operatorname{rwg}(j)$ of the safety climate scale across NICUs was 0.86 (with a range of 0.68 to 0.93 ) further suggesting the appropriateness of aggregation to the NICU level.

\section{Direct and interactive relationships between safety climate and safety climate strength in relation to LOS}

Table 1 shows the characteristics of the clinical sample. Of the 7338 VLBW infants included in the study, 653 (8.9\%) died prior to discharge and 3 had incomplete LOS information, resulting in 6682 infants for the primary analysis. Mean LOS was 68 days, with a median LOS of 60 days (IQR 41 to 88 days).

Table 2 shows patient-level associations with LOS after adjustment for clinical characteristics. All three models are shown, with sequentially increasing complexity including evaluation of safety climate PPR (model 1), safety climate PPR and safety climate strength (model 2), and their interaction (model 3). Safety climate PPR was not associated with LOS in models 1 or 2 , but safety climate strength was associated with LOS in models 2 and 3. Addition of safety climate strength as an interaction term revealed divergent associations as illustrated in Fig. 2, modeled at \pm 1 SD from the mean climate strength. NICUs with weak climate (1 SD below the mean climate strength) and low PPR had LOS 3 days

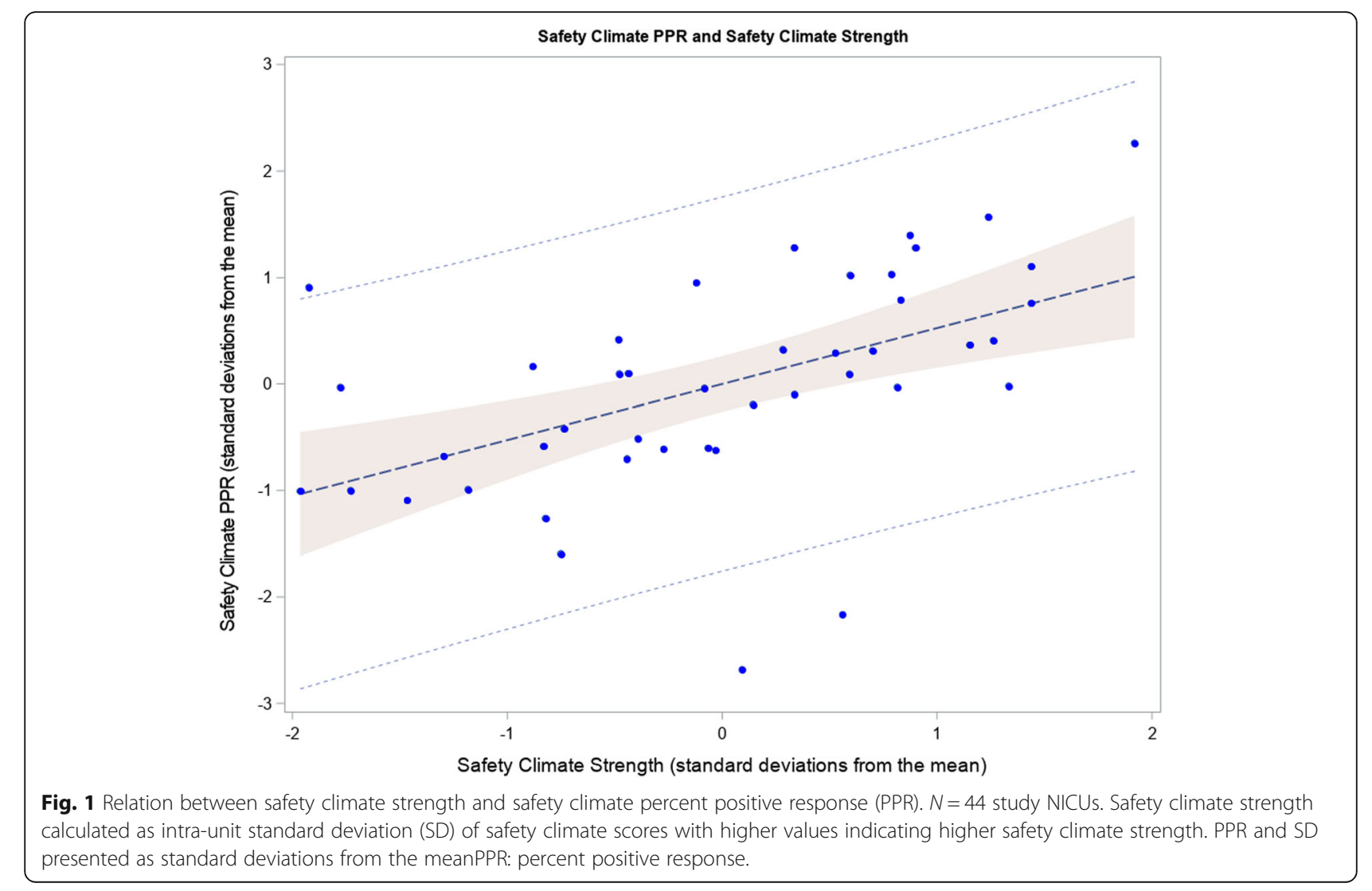


Table 1 Description of survey respondents and clinical sample

\begin{tabular}{|c|c|}
\hline & $\mathrm{N}(\%)$ or mean $( \pm \mathrm{SD})$ \\
\hline NICUs, N & 44 \\
\hline \multicolumn{2}{|l|}{ Level of care } \\
\hline Intermediate & $6(14)$ \\
\hline Community & $27(61)$ \\
\hline Regional & $11(25)$ \\
\hline Survey response rate & $70 \%( \pm 20)$ \\
\hline Safety Climate PPR & $66 \%( \pm 12)$ \\
\hline Safety Climate strength & $17( \pm 3.1)$ \\
\hline Respondents, $N$ & 2073 \\
\hline Females & $1697(85)$ \\
\hline \multicolumn{2}{|l|}{ Typical shift } \\
\hline Days & $894(48)$ \\
\hline Nights/Evenings & $681(36)$ \\
\hline Variable & $293(16)$ \\
\hline \multicolumn{2}{|l|}{ Position } \\
\hline Physician & $235(12)$ \\
\hline Neonatal nurse practitioner & $35(1.7)$ \\
\hline Registered nurse & $1464(72)$ \\
\hline Respiratory therapist & $286(14)$ \\
\hline Other & $21(1.0)$ \\
\hline \multicolumn{2}{|l|}{ Years in specialty } \\
\hline$<1$ & $47(2.4)$ \\
\hline $1-4$ & $266(13)$ \\
\hline $5-10$ & $476(24)$ \\
\hline $11-20$ & $538(27)$ \\
\hline$>=21$ & $643(33)$ \\
\hline VLBW infants, $N$ & 7338 \\
\hline Gestational age, weeks & $28.2( \pm 2.9)$ \\
\hline Birthweight, grams & $1061( \pm 285)$ \\
\hline Small for gestational age & $1392(19)$ \\
\hline Male sex & $3701(50)$ \\
\hline \multicolumn{2}{|l|}{ 5-min APGAR score } \\
\hline$<4$ & $449(6.1)$ \\
\hline $4-6$ & $1298(18)$ \\
\hline$>6$ & $5591(76)$ \\
\hline Inborn & $5686(77)$ \\
\hline Length of stay (all infants), days & $66( \pm 40)$ \\
\hline Length of stay (survivors), days & $68( \pm 37)$ \\
\hline PMA-DC (all infants), weeks & $38( \pm 5.0)$ \\
\hline PMA-DC (survivors), weeks & $39( \pm 3.8)$ \\
\hline Mortality & $653(8.9)$ \\
\hline Chronic lung disease & $1415(25)$ \\
\hline Health care-associated infection & $640(8.7)$ \\
\hline
\end{tabular}

higher than the mean, and each $10 \%$ increase in PPR associated with a 1.73-day decrease in LOS. NICUs with strong climate (1 SD above the mean climate strength) and low PPR had LOS 1 day lower than the mean, and each 10\% increase in PPR associated with a nonsignificant trend toward increase in LOS. Weak NICUs with low PPR had longer LOS than strong NICUs with low PPR $(P<.001)$, but there was no difference between weak and strong NICUs with high PPR $(P=.07)$.

\section{Sensitivity analyses}

The results did not differ materially in sensitivity analyses, shown in Additional file 1: Table S1. These analyses used $r_{w g(j)}$ as the indicator of safety climate strength, used PMA-DC as the marker for LOS, and included patient deaths in the LOS analysis, respectively. Stratification of patient outcomes by birth year is shown in Additional file 1: Figure S2.

\section{Secondary outcome measures}

Figure 3 and Additional file 1: Table S2 show the associations of safety climate PPR and safety climate strength with other clinical outcomes. In a similar pattern to the primary analysis, NICUs with strong climates and low PPR exhibited the lowest odds of risk-adjusted HAI. CLD exhibited no direct effects for PPR or climate strength in the absence of the interaction, but a marginally significant interaction effect. PPR and climate strength were significantly related to mortality directly and in interaction modeling. For both CLD and mortality, higher PPR associated with improved outcomes in interaction modeling, but the climate strength interaction did not translate to clinically meaningful differences.

\section{Discussion}

This study found that safety climate PPR and safety climate strength are associated with LOS among VLBW infants. Specifically, safety climate strength moderates the association between safety climate PPR and LOS among VLBW infants in divergent ways.

Historically, safety climate has been considered a score on a 100-point scale assuming a shared consensus of local emphasis on patient safety. In this direct consensus model, variation in responses is considered a nuisance of imprecise measurement. However, a dispersion model views the variability of responses as a focal construct. (36) Ginsburg and Oore recently recommended a multifaceted approach to safety climate analysis, including safety climate level, safety climate strength, and histogram analysis. (24) Our analysis attempts to apply this approach by combining safety climate level (measured as PPR) and safety climate strength (measured as SD) to explain variation in NICU LOS. 
Table 2 Relationship between safety climate, safety climate strength and length of stay

\begin{tabular}{|c|c|c|c|c|c|}
\hline & Parameter estimate & SE & Incremental F & $P$ & RMSE \\
\hline \multicolumn{6}{|l|}{ Model 1 (CF + PPR) } \\
\hline Safety Climate PPR & -0.38 & 0.31 & 1.52 & .22 & 23.23 \\
\hline \multicolumn{6}{|l|}{ Model 2 (CF + PPR + Strength) } \\
\hline Safety Climate PPR & 0.62 & 0.39 & 2.58 & .11 & 23.20 \\
\hline Safety Climate Strength & -1.59 & 0.37 & 18.59 & $<.001$ & \\
\hline \multicolumn{6}{|c|}{ Model $3(C F+P P R+$ Strength + PPR*Strength) } \\
\hline Safety Climate PPR & 5.20 & 1.79 & 8.48 & .004 & 23.19 \\
\hline Safety Climate Strength & -5.92 & 1.69 & 12.27 & $<.001$ & \\
\hline Safety Climate PPR * Strength & -5.58 & 2.12 & 6.89 & .009 & \\
\hline
\end{tabular}

$n=6682$ infants in 44 NICUs. Ordinary least squares regression analysis at the patient level, with LOS transformed to log-normal scale. $R M S E$ root mean square error, reflecting standard deviation of the unexplained variance. Lower values indicate better model fit. LOS Length of stay, PPR Percent positive response.

CF Clinical factors: sex, gestational age, 5 min Apgar score, small for gestational age, outborn, birth weight, antenatal steroids, fetal distress, major anomalies, maternal hypertension, and maternal race.

Although safety climate PPR and safety climate strength showed moderate association with each other, safety climate strength moderated the association between PPR and LOS in divergent ways. While the interpretation of safety climate PPR is straightforward, the interpretation of safety climate strength merits further consideration. It is possible that two distinct principles affect safety climate strength: (1) the average level of safety climate perceptions within the unit and (2) the true consistency of safety culture underlying the safety climate perceptions.

Due to the ceiling effects with a limited response scale, NICUs with high safety climate PPR are expected to also exhibit agreement among respondents. For instance, upper limits were much more frequently encountered than the lower limits (7.6\% of respondents scored 100/ 100, while no respondents scored 0/100). Consistent with this, high safety climate PPR scores corresponded with less variability among respondents (i.e., stronger safety climate). Conversely, NICUs with lower safety climate PPR showed greater variability in general (i.e., weaker safety climate), as even NICUs with poor aggregate safety climate had some individuals with positive safety climate perceptions. As an illustration, at least one-third of respondents reported positive safety climate in all NICUs, and every NICU had at least one respondent scoring $>95 / 100$.

However, NICUs exhibited differences in safety climate strength not fully explained by the safety climate PPR, suggesting that the strength of a safety

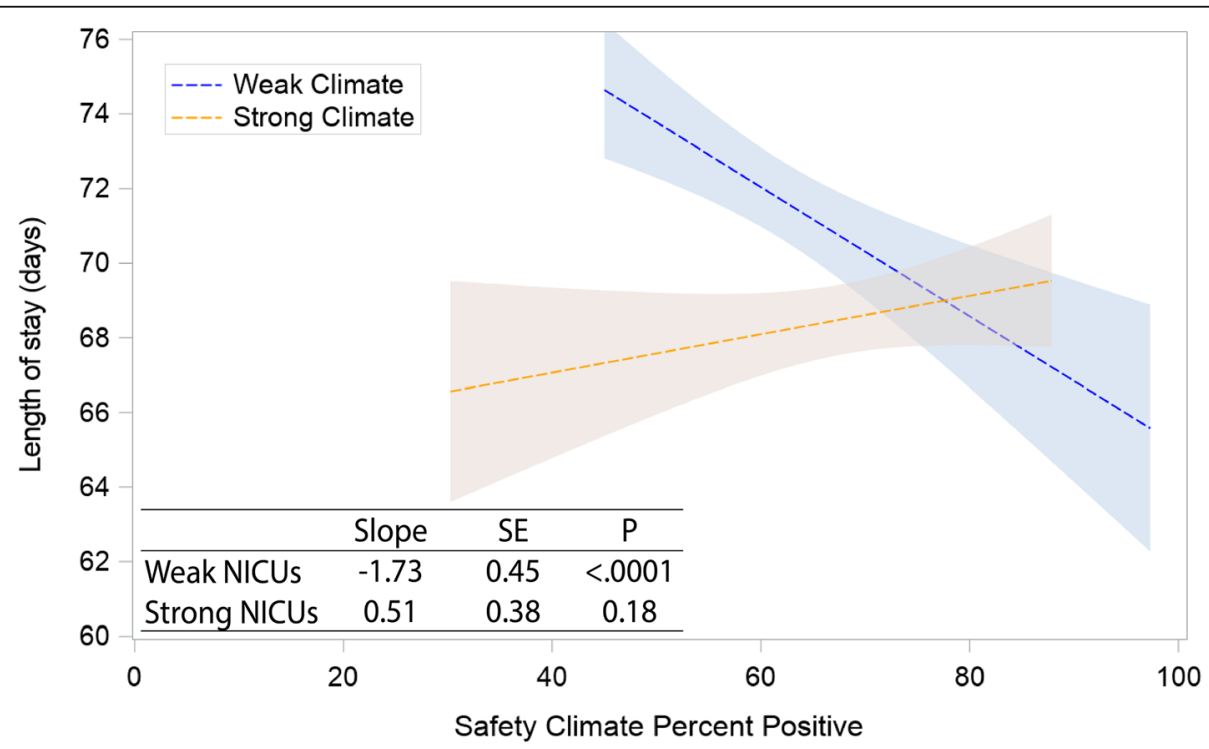

Fig. 2 Safety climate strength and the relation between percent positive response and length of stay. Effect of safety climate strength on the relation between safety climate percent positive response (PPR) and risk-adjusted length of stay among very low birthweight infants 


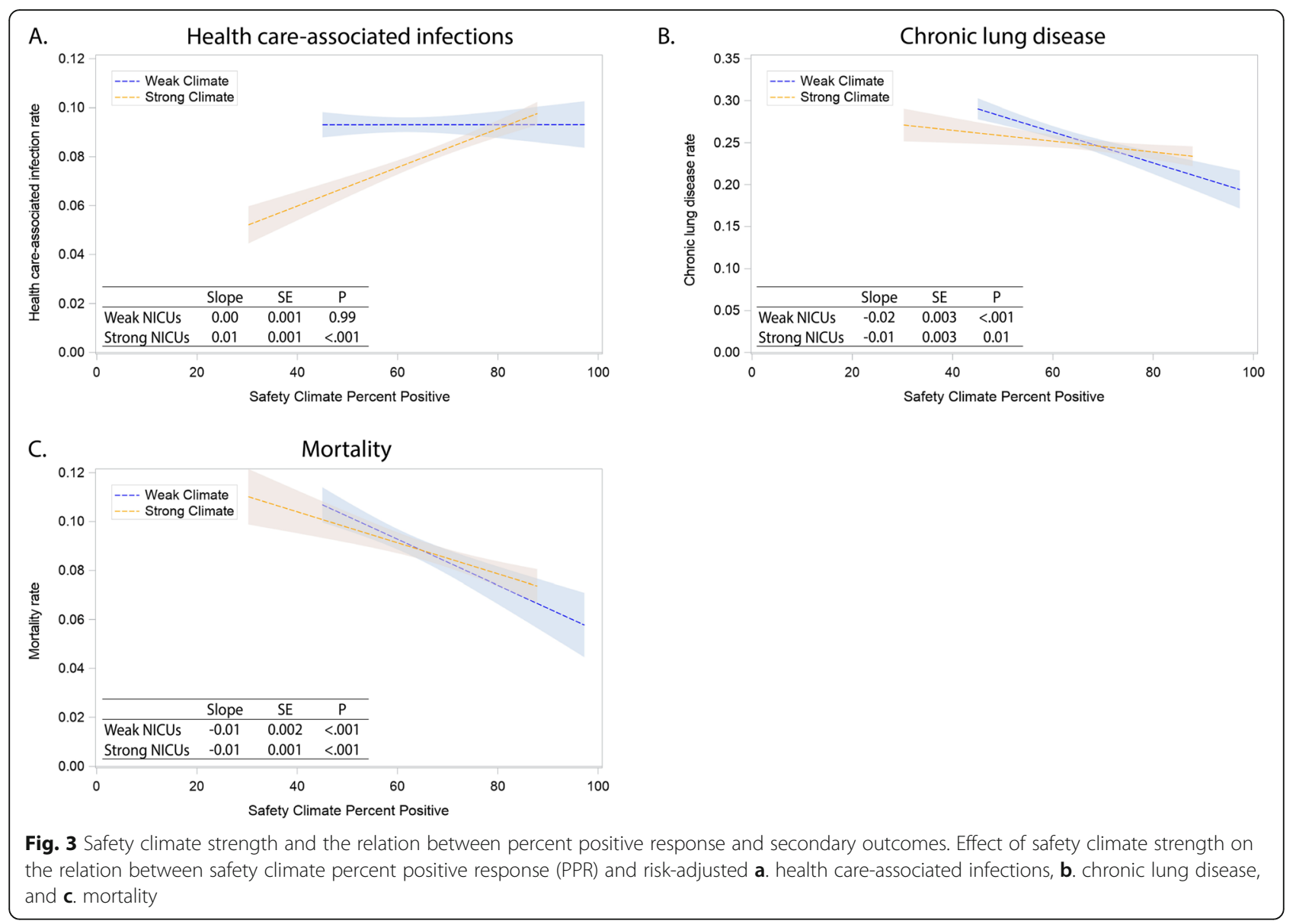

climate does not only correspond to its level. Consistency and convergence of perceptions about safety climate, i.e., safety climate strength, can result from objective and social factors. The shared conditions of the workplace (e.g., actual safety performance) and interdependent work can homogenize perceptions. The process through which this occurs is referred to as social information processing, whereby ongoing social influence and learning occurs through help-seeking and other work-related interactions. (46) Convergent attitudes can cement over time through attraction-selection-attrition processes that increase similarity in the priority placed on safety by favoring selection and retention of employees who value a positive safety climate. (47) For example, a NICU may have inadequate resources to promote safety, and workers may discuss this lack of resources as they carry out their interdependent work. In this scenario, the social information processing at work in the NICU results in both low safety climate PPR and high consensus about the low safety climate, increasing safety climate strength. It is the independent and joint consequences of safety climate PPR and safety climate strength that have been evaluated in this study.
The observed association between safety climate strength and LOS suggests that safety climate not only affects typical safety domains, but also efficiency of care. When appropriately risk-adjusted, LOS serves as a straightforward indicator of composite outcomes. A wide range of adverse events are expected to increase LOS, including medication errors, surgical errors, and HAIs. However, LOS serves as a marker of efficiency beyond mere prevention of errors. In this way, our findings suggest that a strong safety climate may be indicative of strong shared basis for action that facilitates coordination and teamwork among NICU providers, resulting in more safely and swiftly transitioning infants from dependency to discharge. Furthermore, the magnitude of this association is practically significant, with average LOS differences of several days in relation to safety climate strength. For the median-sized NICU in this study, a 1-day reduction in average LOS among VLBW infants translates to 119 fewer patient-days per year, representing hundreds of thousands of health care dollars saved and better resource availability for infants with higher need. (28) Among neonatology quality improvement, reducing LOS of VLBW infants by $1-5$ days is considered a significant improvement, particularly considering that 
LOS is partially dictated by physiological maturity around 34-35 weeks post-menstrual age, limiting the degree of reduction that could be expected. (48-51)

In addition, we find an interaction effect between safety climate PPR and safety climate strength, suggesting the value of considering safety climate strength. Specifically, and unexpectedly, we find NICUs with strong safety climate but low safety climate PPR exhibited the earliest discharges and lowest rates of HAIs. Although contrary to expectations, we speculate that this surprising relationship results from a motivation to improve that may result from the shared perception that we are not safe around here and we all agree on that (i.e., the combination of perceiving low safety climate PPR and strong safety climate). That particular combination means that at least the preponderance of employees perceive a shared reality in which the safety climate is in need of improvement. The NICUs in the present study were participants in a voluntary quality improvement project, and thus may have been especially likely to connect low safety climate PPR with a need for improvement. The corresponding sense of urgency that often accompanies shared perceptions of problematic conditions fosters collective, coordinated behavioral change that can improve outcomes. (52) This sense of urgency may not be present in NICUs with high PPR, even if exhibiting a strong climate. In contrast, low safety climate PPR with weak safety climate means employees may be experiencing the climate in divergent ways and find it difficult to generate appropriate, coordinated responses. Lack of coordination can slow care delivery, increase likelihood of adverse events, and extend LOS.

Although evaluation of a causal link between safety climate strength and quality of care is in its infancy, the characteristics that have been identified as enhancing climate strength are also likely to foster behavior that expedites high quality care delivery and reduces LOS including unit cohesion, stability, and dense communication networks. (53-55) Leader actions also contribute to safety climate strength. Specifically, transformational leadership grounded in higher quality exchanges between leaders and subordinates can generate stronger safety climates and better outcomes through individualized interaction, $(53,56,57)$ providing more information, $(58,59)$ and making priorities clear by offering feedback and recognition for safety-related behaviors. (60) Leaders can also cultivate their ability to enhance safety climate PPR and strength by improving their safety-related interactions through data, feedback, and tools. (59, 61, 62) One such example is Leadership WalkRounds with feedback, which has been shown to have profound impacts on safety climate and provider engagement. $(63,64)$ In summary, when unit members interact more and when leaders communicate more fully to state and reinforce safety priorities, climate strength increases. (26)

It should be noted that although the details varied for the secondary outcomes, safety climate strength still contributed important detail to the interpretation of PPR alone. Individually, these are relatively infrequent outcomes among VLBW infants, and this study was not adequately powered to detect subtle differences in these outcomes. Thus, it remains plausible that safety climate PPR and safety climate strength are associated with these outcomes, but larger scale studies are needed to evaluate this hypothesis.

This study must be interpreted in the context of its design. As a cross-sectional study, our research cannot determine causality of the observed associations and suggests the need for future longitudinal research that measures changes in safety climate over time. Participation in this study was limited and available on a firstcome basis, raising the possibility of response bias at the NICU level. However, such a bias would make it more likely that participating NICUs had high safety climate PPR (and relatively stronger climate), making it less likely to find significant effects. Selection bias among respondents is also possible and we were unable to compare respondent demographics to non-respondent demographics, although our response rate of $63 \%$ compares favorably with acceptable thresholds for response rates and other studies of safety climate, including studies validating the Safety Attitudes Questionnaire. (13, 34, $65,66)$ The study sample also mitigates the concerns of bias as it offers a large, diverse, and representative sample of NICUs across the state of California. As such, the findings are more readily able to generalize to NICUs. Although we have used extensive risk-adjustment in line with prior research, $(29,67)$ LOS is highly dependent on baseline clinical characteristics and it is possible unmeasured confounders remain. As a hypothesisgenerating study, no correction was made for multiple testing. Because our phenomena of interest (safety climate PPR and safety climate strength) occur at the NICU level, we did not use a random effects approach to account generally for unit-level variation, potentially excluding relevant NICU-level effects unrelated to safety climate. However, we did include a wide set of unit and patient-level controls, and employed cluster-robust standard errors to mitigate this risk.

\section{Conclusion}

Prior research in health care has emphasized safety climate PPR largely to the exclusion of the consistency of perceptions of safety climate, i.e., safety climate strength. We find that omission is costly, as safety climate strength has both a direct and moderating effect on LOS among VLBW infants. The specific form of the 
interaction further suggests that for units with low safety climate PPR and low safety climate strength, they might benefit most by building awareness and cultivating consistent assessments as a basis for engagement in patient safety interventions. Evaluations of NICU safety climate should thus diagnose and account for the distribution across respondents. Our findings also demonstrate that promoting safety climates with high PPR and strength within NICUs provides benefits beyond safety to the efficiency of care for these vulnerable patients.

\section{Supplementary information}

Supplementary information accompanies this paper at https://doi.org/10. 1186/s12913-019-4592-1.

Additional file 1: Figure S1. Representative safety climate score distributions for NICUs with stronger and weaker safety climate strengths. Table S1. Relationship between safety climate, safety climate strength, and length of stay. Table S2. Relationship between safety climate and secondary clinical outcomes. Figure $\mathbf{S 2}$. Effect of safety climate strength on the relation between safety climate percent positive response (PPR) and risk-adjusted length of stay among very low birthweight infants, stratified by birth year.

\section{Abbreviations}

CLD: Chronic lung disease; CPQCC: California Perinatal Quality Care Collaborative; HAl: Health care-associated infection; LOS: Length of stay; MD: Physician; NICU: Neonatal intensive care unit; NNPs: Neonatal nurse practitioners; PMA-DC: Post-menstrual age at discharge; PPR: Percent positive response; RN: Registered nurse; SAQ: Safety Attitudes Questionnaire; VLBW: Very low birth weight

\section{Acknowledgements}

Not applicable.

\section{Authors' contributions}

All authors have read and approved the manuscript as submitted. ICMJE criteria for authorship read and met: DST EJT TJV JBL PJS CCN HCL JBS JP. Agree with the manuscript's results and conclusions: DST EJT TJV JBL PJS CCN HCL JBS JP. Designed the experiments/the study: DST, EJT, TJV, JBL, $H C L$, JBS, JP. Analyzed the data: DST, JBL, JP. Wrote the first draft of the paper: DST. Assisted with approach and selection of data inputs: DST, EJT, TJV, JBL, HCL, JBS, JP. Assisted with interpretation of results: DST, EJT, TJV, JBL, PJS, CCN, HCL, JBS, JP. Contributed to revision of the paper: DST, EJT, TJV, $J B L, P J S, C C N, H C L, J B S, J P$.

\section{Funding}

This work was supported by the Eunice Kennedy Shriver National Institute of Child Health and Human Development [R01 HD084679-01, Co-PI: Sexton and Profit and K24 HD053771-01, Pl: Thomas], Texas Children's Hospital [Pediatrics Pilot Research Fund 33-126, PI: Profit] where Dr. Profit worked at the time of this research, and the Jackson Vaughan Critical Care Research Fund. All funding bodies had no role in the study design, data collection, analysis, interpretation of the data, writing the manuscript, or the decision to submit for publication.

\section{Availability of data and materials}

The data that support the findings of this study are available from the California Perinatal Quality Care Collaborative, but restrictions apply to the availability of these data, which were used under license for the current study, and so are not publicly available. Data are however available from the first author (dtawfik@stanford.edu) upon reasonable request and with permission of the California Perinatal Quality Care Collaborative.

\section{Ethics approval and consent to participate}

This study was approved by the Institutional Review Board of Stanford University with waiver of informed consent.

\section{Consent for publication}

Not applicable.

\section{Competing interests}

The authors declare that they have no competing interests.

\section{Author details}

${ }^{1}$ Division of Pediatric Critical Care Medicine, Department of Pediatrics, Stanford University School of Medicine, 770 Welch Road, Suite 435, Stanford, CA 94304, USA. ${ }^{2}$ The McGovern Medical School at The University of Texas Health Science Center at Houston, Houston, TX, USA. ${ }^{3}$ The University of Texas - Memorial Hermann Center for Healthcare Quality and Safety, Houston, TX, USA. ${ }^{4}$ Graduate School of Management, Vanderbilt University, Nashville, TN, USA. ${ }^{5}$ Perinatal Epidemiology and Health Outcomes Research Unit, Division of Neonatology, Department of Pediatrics, Stanford University School of Medicine, Stanford, CA, USA. ${ }^{6}$ California Perinatal Quality Care Collaborative, Stanford, CA, USA. ${ }^{7}$ Center for Quality and Clinical Effectiveness, Lucile Packard Children's Hospital, Palo Alto, CA, USA. ${ }^{8}$ Division of Pediatric Hospitalist Medicine, Department of Pediatrics, Stanford University, Stanford, CA, USA. 'Department of Psychiatry, Duke University Health System, Duke University School of Medicine, Durham, NC, USA.

${ }^{10}$ Duke Center for Healthcare Safety and Quality, Duke University Health System, Durham, NC, USA.

Received: 8 March 2019 Accepted: 9 October 2019

Published online: 22 October 2019

\section{References}

1. Weick KE, Sutcliffe KM. Managing the unexpected: resilient performance in an age of uncertainty. 2nd ed. San Francisco: Jossey-Bass; 2007.

2. Vogus TJ. Safety climate strength: a promising construct for safety research and practice. BMJ Qual Saf. 2016;25(9):649-52.

3. Ausserhofer D, Schubert M, Desmedt M, Blegen MA, De Geest $S$, Schwendimann R. The association of patient safety climate and nurserelated organizational factors with selected patient outcomes: a crosssectional survey. Int J Nurs Stud. 2013;50(2):240-52.

4. Bell BG, Reeves D, Marsden K, Avery A. Safety climate in English general practices: workload pressures may compromise safety. J Eval Clin Pract. 2016;22(1):71-6.

5. Budin WC, Gennaro S, O'Connor C, Contratti F. Sustainability of improvements in perinatal teamwork and safety climate. J Nurs Care Qual. 2014;29(4):363-70.

6. Hessels AJ, Genovese-Schek V, Agarwal M, Wurmser T, Larson EL. Relationship between patient safety climate and adherence to standard precautions. Am J Infect Control. 2016;44(10):1128-32.

7. Hughes LC, Chang Y, Mark BA. Quality and strength of patient safety climate on medical-surgical units. JONA. 2012;42(10 Suppl):S27-36.

8. Leiss JK. Safety climate and use of personal protective equipment and safety medical devices among home care and hospice nurses. Ind Health. 2014:52(6):492-7.

9. Nelson S, Stone PW, Jordan S, Pogorzelska M, Halpin H, Vanneman M, et al. Patient safety climate: variation in perceptions by infection preventionists and quality directors. Interdiscip Perspect Infect Dis. 2011;2011:357121.

10. Profit J, Lee HC, Sharek PJ, Kan P, Nisbet CC, Thomas EJ, et al. Comparing NICU teamwork and safety climate across two commonly used survey instruments. BMJ Qual Saf. 2016;25(12):954-61.

11. Rozenbojm MD, Nichol K, Spielmann S, Holness DL. Hospital unit safety climate: relationship with nurses' adherence to recommended use of facial protective equipment. Am J Infect Control. 2015:43(2):115-20.

12. Santiago $T H$, Turrini RN. Organizational culture and climate for patient safety in Intensive Care Units. Revista da Escola de Enfermagem da U S P. 2015:49:123-30.

13. Sexton JB, Helmreich RL, Neilands TB, Rowan K, Vella K, Boyden J, et al. The safety attitudes questionnaire: psychometric properties, benchmarking data, and emerging research. BMC Health Serv Res. 2006;6:44.

14. Singer SJ, Vogus TJ. Safety climate research: taking stock and looking forward. BMJ Qual Saf. 2013;22(1):1-4. 
15. Turnberg W, Daniell W. Evaluation of a healthcare safety climate measurement tool. J Saf Res. 2008;39(6):563-8.

16. Tsao K, Browne M. Culture of safety: A foundation for patient care. Semin Pediatr Surg. 2015;24(6):283-7.

17. Singer SJ, Vogus TJ. Reducing hospital errors: interventions that build safety culture. Annu Rev Public Health. 2013;34:373-96.

18. Sexton JB, Berenholtz SM, Goeschel CA, Watson SR, Holzmueller CG, Thompson DA, et al. Assessing and improving safety climate in a large cohort of intensive care units. Crit Care Med. 2011;39(5):934-9.

19. Hansen LO, Williams MV, Singer SJ. Perceptions of hospital safety climate and incidence of readmission. Health Serv Res. 2011;46(2):596-616.

20. Comprehensive accreditation manual for hospitals: The Joint Commission; 2016.

21. Sorra J, Famolaro T, Dyer N. Hospital Survey on Patient Safety Culture: 2012 User Comparative Database Report (Prepared by Westat, Rockville, MD, under contract No. HHSA 290200710024C). AHRQ Publication No. 12-0017; 2013.

22. Group TL. Guidelines for a culture of safety survey [Available from: http:// www.leapfroggroup.org/sites/default/files/Files/Guidelines_CultureSurvey_2 0170401_0_0.pdf.

23. Meddings J, Reichert $\mathrm{H}$, Greene MT, Safdar N, Krein SL, Olmsted RN, et al. Evaluation of the association between hospital survey on patient safety culture (HSOPS) measures and catheter-associated infections: results of two national collaboratives. BMJ Qual Saf. 2017;26(3):226-35.

24. Ginsburg L, Gilin OD. Patient safety climate strength: a concept that requires more attention. BMJ Qual Saf. 2016;25(9):680-7.

25. Mischel W. Personality and assessment: Wiley; 1968. p. 365

26. Schneider B, Ehrhart MG, Macey WH. Organizational climate and culture. Annu Rev Psychol. 2013;64:361-88.

27. Kohn LT, Corrigan JM, Donaldson MS. To err is human: building a safer health system. Washington: National Academy Press; 2000.

28. Zupancic J. A systematic review of costs associated with preterm birth. In: Behrman RE, Butler AS, editors. Preterm birth: causes, consequences, and prevention. Washington: National Academies Press; 2007.

29. Lee HC, Bennett MV, Schulman J, Gould JB, Profit J. Estimating length of stay by patient type in the neonatal intensive care unit. Am J Perinatol. 2016;33(8):751-7.

30. Profit J, Etchegaray J, Petersen LA, Sexton JB, Hysong SJ, Mei M, et al. Neonatal intensive care unit safety culture varies widely. Arch Dis Child Fetal Neonatal Ed. 2012;97(2):F120-6

31. Profit J, Etchegaray J, Petersen LA, Sexton BJ, Mei M, Thomas EJ. Safety attitudes among neonatal intensive care units vary widely. E-PAS. 2010;4425:595.

32. Gould JB. The role of regional collaboratives: the California perinatal quality care collaborative model. Clin Perinatol. 2010;37(1):71-86

33. Profit J, Etchegaray J, Petersen LA, Sexton JB, Hysong SJ, Mei M, et al. The safety attitudes questionnaire as a tool for benchmarking safety culture in the NICU. Arch Dis Child Fetal Neonatal Ed. 2012;97(2): F127-32.

34. Shanafelt TD, Mungo M, Schmitgen J, Storz KA, Reeves D, Hayes SN, et al. Longitudinal study evaluating the association between physician burnout and changes in professional work effort. Mayo Clin Proc. 2016; 91(4):422-31.

35. Profit J, Zupancic JA, Gould JB, Pietz K, Kowalkowski MA, Draper D, et al. Correlation of neonatal intensive care unit performance across multiple measures of quality of care. JAMA Pediatr. 2013;167(1):47-54.

36. Schneider B, Salvaggio AN, Subirats M. Climate strength: a new direction for climate research. J Appl Psychol. 2002;87(2):220-9.

37. Zohar D, Luria G. A multilevel model of safety climate: cross-level relationships between organization and group-level climates. J Appl Psychol. 2005;90(4):616-28.

38. Horbar JD, Soll RF, Edwards WH. The Vermont Oxford network: a Community of Practice. Clin Perinatol. 2010;37(1):29-47.

39. Eichenwald EC, Blackwell M, Lloyd JS, Tran T, Wilker RE, Richardson DK. Inter-neonatal intensive care unit variation in discharge timing: influence of apnea and feeding management. Pediatrics. 2001;108(4): 928-33.

40. Hair AB, Bergner EM, Lee ML, Moreira AG, Hawthorne KM, Rechtman DJ, et al. Premature infants 750-1,250 g birth weight supplemented with a novel human Milk-derived cream are discharged sooner. Breastfeed Med. 2016;11:133-7.
41. Lindell MK, Brandt CJ. Climate quality and climate consensus as mediators of the relationship between organizational antecedents and outcomes. J Appl Psychol. 2000;85(3):331-48.

42. Bliese P, Halverson RR. Group consensus and psychological well-being: a large field study. J Appl Soc Psychol. 1998;28:563-80.

43. Bliese P. Within-group agreement, non-independence, and reliability: implications for data aggregation and analysis. In: Klein KJ, Kozlowski SWJ, editors. Multilevel theory, research, and methods in organization. San Francisco: Jossey-Bass; 2000. p. 349-81.

44. James LR, Demaree RG, Wolf G. Estimating within-group interrater reliability. J Appl Psychol. 1984;69:85-98.

45. Duan N. Smearing estimate - a nonparametric retransformation method. J Am Stat Assoc. 1983;78(383):605-10.

46. Salancik GR, Pfeffer J. A social information processing approach to job attitudes and task design. Adm Sci Q. 1978;23(2):224-53.

47. Schneider B. The people make the place. Pers Psychol. 1987:40(3):437-53.

48. Profit J, Zupancic JA, McCormick MC, Richardson DK, Escobar GJ, Tucker J, et al. Moderately premature infants at Kaiser Permanente medical care program in California are discharged home earlier than their peers in Massachusetts and the United Kingdom. Arch Dis Child Fetal Neonatal Ed. 2006;91(4):F245-50.

49. Lee HC, Bennett MV, Crockett M, Crowe R, Gwiazdowski SG, Keller H, et al. Comparison of Collaborative Versus Single-Site Quality Improvement to Reduce NICU Length of Stay. Pediatrics. 2018;142(1):e20171395.

50. Welch CD, Check J, O'Shea TM. Improving care collaboration for NICU patients to decrease length of stay and readmission rate. BMJ Open Qual. 2017;6(2):e000130.

51. Mansfield SA, Ryshen G, Dail J, Gossard M, McClead R, Aldrink JH. Use of quality improvement (QI) methodology to decrease length of stay (LOS) for newborns with uncomplicated gastroschisis. J Pediatr Surg. 2018;53(8):1578-83.

52. Kotter JP. Leading Change. Boston: Harvard Business School Press; 1996. 187 p.

53. Luria G. Climate strength--how leaders form consensus. Leadersh Q. 2008; 19(1):42-53.

54. Beus JM, Bergman ME, Payne SC. The influence of organizational tenure on safety climate strength: a first look. Accid Anal Prev. 2010;42(5):1431-7.

55. Zohar D, Tenne-Gazit O. Transformational leadership and group interaction as climate antecedents: a social network analysis. J Appl Psychol. 2008;93(4):744-57

56. Zohar D. The effects of leadership dimensions, safety climate, and assigned priorities on minor injuries in work groups. J Organ Behav. 2002;23(1):75-92.

57. Zohar D, Luria G. Group leaders as gatekeepers: testing safety climate variations across levels of analysis. Appl Psychol. 2010;59(4):647-73.

58. Gonzalez-Roma V, Peiro JM, Tordera N. An examination of the antecedents and moderator influences of climate strength. J Appl Psychol. 2002;87(3):465-73.

59. Zohar D, Luria G. Climate as a social-cognitive construction of supervisory safety practices: scripts as proxy of behavior patterns. J Appl Psychol. 2004; 89(2):322-33.

60. Luria G, Zohar D, Erev I. The effect of workers' visibility on effectiveness of intervention programs: supervisory-based safety interventions. J Saf Res. 2008; 39(3):273-80.

61. Zohar D, Polachek T. Discourse-based intervention for modifying supervisory communication as leverage for safety climate and performance improvement: a randomized field study. J Appl Psychol. 2014;99(1):113-24.

62. Zohar D, Werber YT, Marom R, Curlau B, Blondheim O. Modifying head nurse messages during daily conversations as leverage for safety climate improvement: a randomised field experiment. BMJ Qual Saf. 2017;26(8):653-62.

63. Sexton JB, Adair KC, Leonard MW, Frankel TC, Proulx J, Watson SR, et al. Providing feedback following leadership WalkRounds is associated with better patient safety culture, higher employee engagement and lower burnout. BMJ Qual Saf. 2018;27(4):261-70.

64. Sexton JB, Sharek PJ, Thomas EJ, Gould JB, Nisbet CC, Amspoker AB, et al. Exposure to leadership WalkRounds in neonatal intensive care units is associated with a better patient safety culture and less caregiver burnout. BMJ Qual Saf. 2014;23(10):814-22.

65. Zenere A, Zanolin ME, Negri R, Moretti F, Grassi M, Tardivo S. Assessing safety culture in NICU: psychometric properties of the Italian version of safety attitude questionnaire and result implications. J Eval Clin Pract. 2016; 22(2):275-82. 
66. Schutt RK. Investigating the social world: the process and practice of research. 9th ed. Boston: SAGE Publications; 2019.

67. Lee HC, Bennett MV, Schulman J, Gould JB. Accounting for variation in length of NICU stay for extremely low birth weight infants. J Perinatol: official journal of the California Perinatal Association. 2013;33(11):872-6.

\section{Publisher's Note}

Springer Nature remains neutral with regard to jurisdictional claims in published maps and institutional affiliations.

- fast, convenient online submission

- thorough peer review by experienced researchers in your field

- rapid publication on acceptance

- support for research data, including large and complex data types

- gold Open Access which fosters wider collaboration and increased citations

- maximum visibility for your research: over $100 \mathrm{M}$ website views per year

At $\mathrm{BMC}$, research is always in progress. 